\title{
Magnetic excitation spectrum of dimerized antiferromagnetic chains
}

\author{
Götz S. Uhrig and H.J. Schulz \\ Laboratoire de Physique des Solides, Université Paris-Sud, 91405 Orsay, France
}

(October 30, 2018)

\begin{abstract}
Motivated by recent measurements on $\mathrm{CuGeO}_{3}$ the spectrum of magnetic excitations of an antiferromagnetic $S=\frac{1}{2}$ chain with alternating coupling strength is investigated. Wave vector dependent magnons and a continuum with square root behavior at the band edges are found. The spectral density of the continua is calculated. Spin rotation symmetry fixes the gap of the continuum to be twice the elementary magnon gap. This is in excellent agreement with experimental results. In addition, the existence of bound states of two magnons is predicted: below the continuum a singlet and a triplet, above the continuum an "anti-bound" quintuplet. The results are based on field theoretic arguments, RPA calculations, and consideration of the limit of strong alternation.
\end{abstract}

75.40.Gb, 75.10.Jm, 75.50.Ee

One-dimensional quantum antiferromagnets can exhibit the interesting phenomenon of the spin-Peierls transition, i.e. a spontaneous dimerization of the lattice and the exchange constant. The recent discovery [1] of a spin-Peierls transition in the inorganic compound $\mathrm{CuGeO}_{3}$ for the first time made neutron scattering investigations in the ordered state possible. These provide interesting information on the dynamic spin correlations and therefore on the magnetic excitations.

The neutron scattering experiments exhibit a number of interesting features: the magnon line shape asymmetry [5.6] observed experimentally and the interpretation of the behavior of the equal-time structure factor [5] indicate quite clearly the existence of a continuum besides a dominant magnon peak. Its weight is roughly equivalent to the one in the main peak [5]. Very recent neutron scattering measurements with high resolution [7] show a "double gap" structure: at $k=\pi$ one finds beside a magnon peak at the gap $\omega=\Delta$ another gap which separates the magnon from the band edge of the continuum. It turns out that this second gap equals within experimental accuracy the first one, i.e. the onset of the continuum is at $2 \Delta$. Furthermore, there is presently an active debate on the importance of next-nearest neighbor couplings 810 . Evidence for a finite competing nextnearest neighbor coupling stems from fits of the magnetic susceptibility [8.9] $\chi(T)$ and from a fit of the equal-time structure factor.

These points motivate the present work. Its aim is to clarify theoretically the magnetic excitation spectrum in the dimerized, spin-Peierls phase below the transition temperature $T_{\mathrm{SP}} \approx 14.3 \mathrm{~K}$ in the dimerized phase.

In the dimerized phase it is reasonable to adopt an adiabatic approach toward the coupling between the magnetic and the phonon subsystem [8 11]. This is possible since the phonon subsystem is three dimensional and hence its fluctuations are weak. This holds in particular well below the transition temperature. It seems furthermore legitimate to neglect to first approximation the interchain coupling [2, 5].

For the above reasons we will focus on the following Hamiltonian

$$
\hat{H}=J \sum_{i}\left[\left(1+(-1)^{i} \delta\right) \boldsymbol{S}_{i} \cdot \boldsymbol{S}_{i+1}+\alpha \boldsymbol{S}_{i} \cdot \boldsymbol{S}_{i+2}\right],
$$

where the $\boldsymbol{S}_{i}$ are spin $-1 / 2$ operators. Beside nearestneighbor coupling $J>0$, next-nearest neighbor coupling $\alpha J$ has been included. The values of $\alpha$ used so far range between 0.24 and 0.36 [8] 10 . We now go to a fermionic representation of the physics of the model via the JordanWigner transformation 12 to fermionic degrees of freedom described by operators $c_{i}, c_{i}^{\dagger}$. One then has $S_{i}^{z}=$ $n_{i}-1 / 2, n_{i}=c_{i}^{\dagger} c_{i}$, and $S_{i}^{+}=c_{i}^{\dagger} \prod_{j=-\infty}^{i}\left(1-2 n_{i}\right)$, and for $\alpha=0$ one obtains

$$
\begin{aligned}
\hat{H} & =\frac{J}{2} \sum_{i}\left(1+(-1)^{i} \delta\right) \\
& \times\left[-c_{i}^{\dagger} c_{i+1}-c_{i+1}^{\dagger} c_{i}+2\left(\frac{1}{2}-n_{i}\right)\left(\frac{1}{2}-n_{i+1}\right)\right] .
\end{aligned}
$$

For $\alpha \neq 0$ next-nearest neighbor terms are also present. For $\delta=0$ the properties of this model are wellunderstood, mainly thanks to Bethe's exact solution [13 16. In particular, there are no spin-wave excitations, but the low-lying excitations rather form a spinon continuum with lower bound $(\pi J / 2) \sin k$.

We now note that in the dimerized phase the momentum $k$ is not conserved: any mode at $k$ is coupled to the mode $k+\pi$. If $\omega$ belongs to the spectrum at $k$, it does so at $k+\pi$ as well. Together with the inversion symmetry $k \leftrightarrow-k$ one obtains thus reflection symmetry about $k=\pi / 2$. This symmetry is not taken into account in previous results [10,11. The reflection symmetry, however, only refers to the energies, not to the spectral weights.

We first consider the case of weak dimerization, $\delta \ll 1$. Then a continuum approximation for the fermionic model (2) is appropriate, and one obtains the Hamiltonian of the so-called massive Thirring model, for which there exists an exact Bethe ansatz solution [17]. The model describes relativistic fermions with "light velocity" $v=\pi J / 2$ and a bare mass $m_{0} \propto \delta$. In addition there is a fermionfermion interaction of strength $g$. The known scaling relation [18] for the magnetic gap (the renormalized mass in fermionic language), $\Delta \propto \delta^{2 / 3}$ (up to logarithmic 
corrections) uniquely fixes $g=2 v$. The exact solution provides valuable information about the spectrum: first there are single-particle and single-hole excitations which in the spin language correspond to states with $\Delta M_{z}= \pm 1$, with excitation energy $\sqrt{\Delta^{2}+v^{2} k^{2}}$. From the fermionic standpoint it is then clear that there is a particle-hole continuum $\left(\Delta M_{z}=0\right)$ with minimum energy $2 \Delta$. However, due to the presence of interactions in the model, there is a well-defined exciton-like particlehole bound state below this continuum [17], at energy $\Delta$ when $g=2 v$. The particle, hole, and exciton thus form the low-energy degenerate triplet excitation (the magnon) expected because of spin rotation invariance of the original lattice model [19]. An applied magnetic field would split this triplet into its three components. In addition, there is another excitonic bound state at energy $\sqrt{3} \Delta / 2[17$. This, however, has no counterpart in the $\Delta M_{z}= \pm 1$ sectors, and therefore is a singlet excitation. Contrary to what was proposed in ref. 11] it is thus not possible to continuously connect (in $k$-space) the two bound state excitations. We note that in a bosonized picture of the fermionic theory 15,16, the particle, hole, and exciton correspond to soliton, antisoliton and breather modes. Also, including a finite $\alpha$ affects the values of $v$ and $\Delta$ quantitatively, but does not affect the general structure of the spectrum which is protected by spin rotation invariance.

From the elementary triplet at energy $\Delta$ it is possible to create multiple excitation continua. Exciting two of the triplet modes, nine different types of states can be created which form states with total spin $S=0,1$, and 2 and minimal energy $2 \Delta$. Only the $S=1$ state is of course accessible in a low-temperature neutron scattering experiment. It is interesting to consider what happens when the dimerization and therefore the gap go to zero. Then $n$-magnon continua, with lower edge at $n \Delta$, become close to the 2-magnon continuum and they all coincide when $\Delta \rightarrow 0$, giving rise to the well-known [14] spinon continuum of the homogeneous spin-1/2 antiferromagnet. One should however notice that the elementary constituents of the continua are $S=1$ objects when $\delta \neq 0$, whereas they are $S=1 / 2$ spinons (or delocalized domain walls) for $\delta=0$. In fact, from the bosonic representation [15, 16 it is clear that a nonzero $\delta$ acts as a linear confining potential between two spinons, i.e. spinons can not exist as elementary excitations when $\delta \neq 0$.

From the above discussion the experimental observation of a $S=1$ continuum at twice the energy of the elementary magnon [7] seems very natural. To investigate the spectral shape of the continuum further we undertook a RPA study at zero temperature of the longitudinal spin correlation function (the density correlation function in fermionic language), starting from eq.(2). The general picture is the same as that of the continuum limit: there is a gap $\Delta$ in the single-particle(-hole) excitation, a particle-hole continuum above $2 \Delta$, and an excitonic bound state within this gap. The existence of the gap for $\delta>0$ helps the approximation by providing an infrared cutoff, i.e. by suppressing low-energy particlehole excitations. For isolated dimers $(\delta \rightarrow 1, \alpha=0)$, the RPA is exact. Yet in general the approximation breaks the spin rotational invariance since the $S^{z}$ component is dealt with differently than the $S^{x}$ and $S^{y}$ components. In a heuristic approach, this can be remedied by choosing an effective interaction constant $J \rightarrow s J$ such that the exciton (magnon) is in the middle of the two-particle continuum gap. Since the static Fock diagram renormalizes the hopping one works in addition with an effective energy scale $J_{\text {eff }}$ and an effective alternation $\delta_{\text {eff }}$. They are both enhanced in comparison to the bare ones.
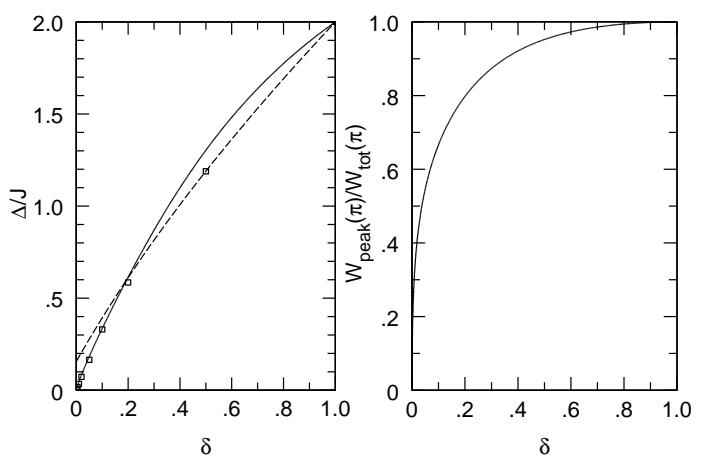

FIG. 1. Left: Gap $\Delta$ vs. alternation $\delta$ compared to perturbative [20] (dashed line) and numerical results (circles) 21] at $\alpha=0$. Right: relative magnon weight at $k=\pi$ vs. $\delta$ at $\alpha=0$.

In fig. 1, results are shown for the alternation dependence of the gap $\Delta(\delta)$ and the weight of the magnon $W_{\text {peak }}(k)$ at $k=\pi$ relative to the total weight, i.e. peak plus continuum. The results at $\alpha=0$ are compared to the perturbation results of Harris 20] and numerical data of Soos et al. [21]. The agreement in the region of interest $\left(\delta \approx 0.03-0.06\right.$ for $\left.\mathrm{CuGeO}_{3}\right)$ is reassuring to proceed along this line. Only for very low $\delta<0.01$ our results become incompatible with the the asymptotics [18,22 $\Delta \propto \delta^{2 / 3} / \sqrt{|\ln (\delta)|}$. The right part of fig. 1 1 shows the spectral weight, which at $\delta=0$ is completely in the diverging continuum 15,23 . It is shifted very quickly into the magnon peak on dimerization.

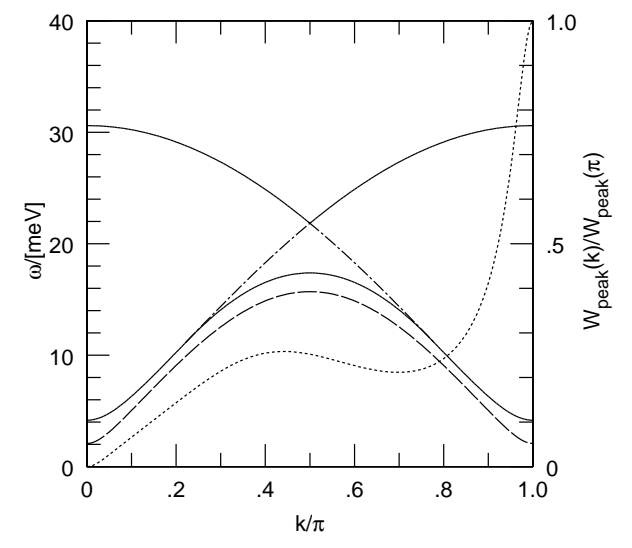


FIG. 2. Dashed curve: magnon dispersion; dotted curve: relative magnon weight (right scale); continuum between solid lines; dashed-dotted lines: intermediate singularity. Numerical values: $\delta_{\text {eff }}=0.136, J_{\text {eff }}=15.3 \mathrm{meV}$ corresponding to $\delta=0.0506, J=11.2 \mathrm{meV}$.

In fig. 2, the RPA spectrum is presented. The longdashed line is the magnon dispersion. The magnon weight relative to its value at $k=\pi$ is depicted by the dotted line. Note its non-monotonic behavior. The continuum is found between the two solid lines. For $k<\pi / 2$ noticeable continuum weight is found only between the lower solid line and the dashed-dotted line. The dasheddotted lines show the positions of a singularity between the band edges. It results from the local maximum at $q=\pi / 2$ of the particle-hole energy $\varepsilon_{\mathrm{ph}}(q)=\varepsilon(k / 2+$ $q)+\varepsilon(k / 2-q)$ where $\varepsilon(q)=J_{\text {eff }} \sqrt{\delta_{\text {eff }}^{2} \sin ^{2}(q)+\cos ^{2}(q)}$. This maximum is not the global one for all $k$ satisfying $\cos (k)<\left(1-\delta_{\text {eff }}\right) /\left(1+\delta_{\text {eff }}\right)$.

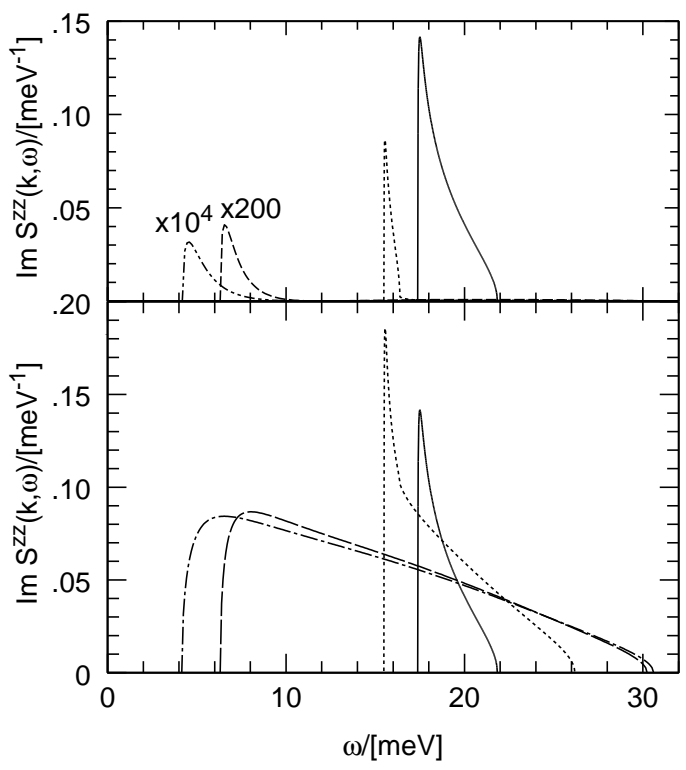

FIG. 3. Continua for different wave vectors, the magnon $\delta$-peaks are not shown. From left to right: upper part $k / \pi=0.01,0.1,0.35,0.5$; lower part $k / \pi=1.0,0.9,0.65,0.5$.

The spectral densities are shown in fig. 3. All singularities (band edges and intermediate) are of square root type. The continua at low $k$ are extremely weak, but extend rigorously speaking up to the same high energies as do their counterparts at $\pi-k$. For $k=(0.5 \pm 0.15) \pi$ the intermediate singularity (cf. fig. 2) is clearly seen at $\omega \approx 17 \mathrm{meV}$. While for $k>\pi / 2$ there is still considerable weight above the intermediate singularity the spectral density for $k<\pi / 2$ practically vanishes. The extreme asymmetry of the continua at $k=\pi / 2$ and $k=(0.5 \pm 0.15) \pi$ can be viewed as the precursor of another sharp resonance which separates from the lower continuum edge in RPA for slightly larger alternation values $(\delta>0.082)$. This is a bound state of two magnons.
The physical origin of this second peak can be understood best in the limit $\delta \rightarrow 1$. Defining $\lambda=(1-\delta) /(1+\delta)$ and setting $J(1+\delta)=1$ a perturbation expansion around complete dimerization can be performed. To assess the influence of the NNN coupling $\bar{\alpha}=\alpha / \lambda$ is kept constant so that $\lambda=0$ still corresponds to isolated dimers. These have $k$-independent triplet excitations at $\omega=1$. The corresponding resolvent $R(k, \omega)=$ $\left\langle S^{z}(k)(\omega-L)^{-1} S^{z}(k)\right\rangle$ is $(1-\cos (k)) /(4(\omega-1))$. Here $L$ is the Liouville operator [24], which stands for commutation with $\hat{H}$. For $\lambda>0$ this mode acquires a finite dispersion since the triplets can hop from dimer to dimer with amplitude $-1 / 4$. In leading order the magnon peak becomes $R_{\text {peak }}(k, \omega)=p_{0} /(\omega-\omega(k))$ with $4 p_{0}=1-\cos (k)-\lambda(\cos (k)+2 \bar{\alpha} \cos (2 k)) / 4$ and $\omega(k)=1-\lambda(1-2 \bar{\alpha}) \cos (2 k) / 2$. For $\bar{\alpha}=0$, Harris calculated $\omega(k)$ in third order [20] in $\lambda$. Note that $\bar{\alpha}>0$ reduces the triplet hopping.

A finite $\lambda$ couples the one-triplet subspace also to the $S=1$ two-triplet subspace. This leads in order $\lambda^{2}$ to the continuum. By a Mori-Zwanzig calculation [24] the corresponding spectral density is found in leading order to be

$$
R_{\mathrm{cont}}(k, \omega)=\frac{\lambda}{4} \frac{(1-\cos (k))(1-2 \bar{\alpha} \cos (k))^{2}}{y+a_{1}-\operatorname{sgn}(y) \sqrt{y^{2}-t^{2}}}
$$

where $a_{1}=(1+2 \bar{\alpha}) / 2, t=(1-2 \bar{\alpha})|\cos (k)|$ and $y$ is the rescaled energy $\omega=2+\lambda y$. The spectral density is finite for $y^{2}<t^{2}$ where the square root in (3) becomes $i \sqrt{t^{2}-y^{2}}$. Thus the continuum is $\propto \lambda \sqrt{t^{2}-y^{2}} /\left(t^{2}+\right.$ $\left.a^{2}+2 a y\right)$. This asymmetric form is qualitatively similar to the results in fig. 3. The edge singularities are square roots. Since no phase transition is expected for $\lambda \in(0,1)$ this supports the RPA result of square root behavior for all finite alternation values in contrast to a previous prediction of a divergence [11].

The positive value of $a_{1}$ is the signature of attraction between two triplets. A $S^{z}=0$ triplet couples to the linear combination of two antiparallel $S^{z}=1$ and $S^{z}=$ -1 triplets. On adjacent dimers they gain $\lambda a / 2$ in energy. For $a_{1}>t$ a bound state emerges which is favored by $\bar{\alpha}>0$. Its energy is found at the zero of the denominator of (3). Since $t$ vanishes at $k=\pi / 2$ the bound state is most prominent at this value. For $\bar{\alpha}=0$ the bound state exists for $k \in[\pi / 3,2 \pi / 3]$; for $\bar{\alpha}>1 / 6$ it is present at all $k$.

Similarly one can investigate the two-triplet dynamics in the $S=0,2$ subspaces. The secular equation found equals the denominator of (3) with interactions $a_{2}=-a_{1}$ for the quintuplet and $a_{0}=2 a_{2}$ for the singlet. This implies an anti-bound quintuplet above the continuum and a more strongly bound singlet below the triplet state for all $k$ even at $\bar{\alpha}=0$. The singlet and the quintuplet where also found at $\bar{\alpha}=0$ by Harris [20]. The attraction for the triplet was missed presumably due to a sign error. It is natural to identify this singlet with the $\sqrt{3} \Delta / 2$ singlet found in the continuum description above. At $\bar{\alpha}=0$ and 
$k=0, \pi$ the singlet lies at the lower continuum band edge which is confirmed numerically 25 for a wide range of $\delta$.

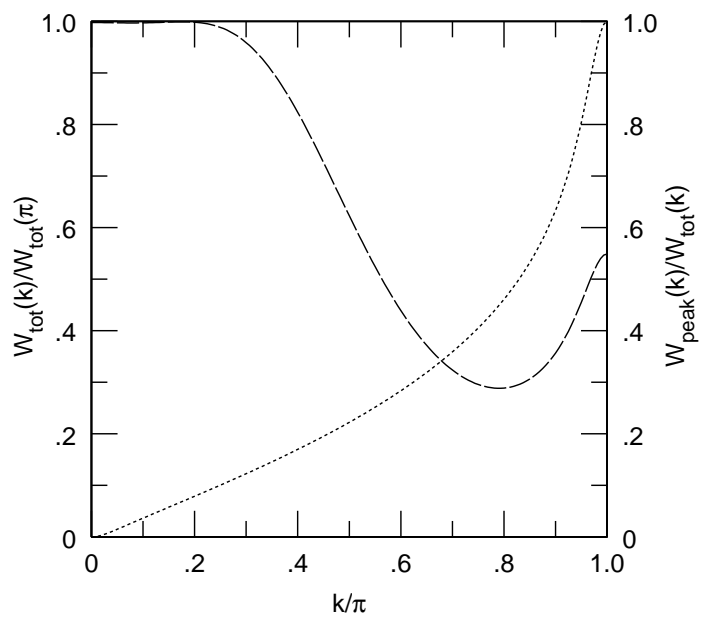

FIG. 4. Dashed curve: magnon weight relative to total weight, right scale; dotted curve: total weight normalized by its value at $k=\pi$, left scale.

Reassured by the coherence of the results of the different approaches we present in fig. 4 the $k$ dependence found in RPA of $W_{\text {tot }}(k)$, proportional to the equal-time structure factor $S(k)$, and of $W_{\text {peak }}(k)$. The agreement with the experimental data [5] is very good. It was previously stressed [10] that $\alpha \approx 1 / 4$ is necessary to achieve agreement whereas the data in fig. 1 is computed at $\alpha=0$. This suggests that $S(q)$ depends mainly on the gap $\Delta$ and not so much on $\alpha$ if $\Delta$ is kept constant by tuning $\delta$. But the interpretation requires care since the approximation tends to raise $W_{\text {tot }}(k)$ around $\pi / 2$. Recall in this context the experimental fit result $\delta=0.042$ which is between our value $(\delta=0.0506)$ and the one used so far 810 . The fact that for $k / \pi$ between 0.6 and 0.9 the spectral weight is found mainly in the continuum is important for the experimental determination of $S(k)[5]$.

Summarizing, we give general arguments for the continuum onset at twice the magnon gap in excellent agreement with experiment [7]. In RPA and for $\delta \rightarrow 1$ we find a square root singularity at the continuum edge which is also in agreement with experiment [7]. Detailed results for the peak weights and the form of the continua are presented. We find evidence in the whole range of $\delta>0$ for a singlet excitation below the continuum for all $k$. In addition, we predict triplet (quintuplet) excitations below (above) the continuum around $k=\pi / 2$ for not too small $\delta$. Their (anti)binding tendency is strongly enhanced by competing NNN coupling. An experimental identification of either of these excitations would be particularly interesting.

We note that in ref. [7] the continuum is attributed to solitonic excitations of the dimerization pattern. However, one then has a important deformation of the crystal lattice and would expect therefore a considerable increase of the effective mass of these objects. The fact that the experimental energy scale of the continuum is the bare $J$ favors a purely magnetic origin for the continuum. Also, in the three-dimensionally ordered structure below $T_{\mathrm{SP}}$ solitons on individual chains accompanied by lattice deformations are confined by the interaction with adjacent chains and therefore cannot be considered as free excitations.

The authors like to thank M. Aïn, L. P. Regnault, S. Haas, and E. Dagotto for communicating their data. This work was supported by the DFG (GSU, individual grant and SFB 341) and by the EEC grant ERBCHRXCT 940438.

[1] M. Hase, I. Terasaki, and K. Uchinokura, Phys. Rev. Lett. 70, 3651 (1993).

[2] M. Nishi, O. Fujita, and J. Akimitsu, Phys. Rev. B 50, 6508 (1994).

[3] J. P. Pouget et al., Phys. Rev. Lett. 72, 4037 (1994).

[4] K. Hirota et al., Phys. Rev. Lett. 73, 736 (1994).

[5] L. P. Regnault et al., Phys. Rev. B 53, 5579 (1996).

[6] M. C. Martin et al., preprint Brookhaven National Laboratory, cond-mat/9603174.

[7] M. Aïn et al., preprint Laboratoire Léon Brillouin, 1996.

[8] J. Riera and A. Dobry, Phys. Rev. B 51, 16098 (1995).

[9] G. Castilla, S. Chakravarty, and V. J. Emery, Phys. Rev. Lett. 75, 1823 (1995).

[10] S. Haas and E. Dagotto, Phys. Rev. B 52, R14396 (1995).

[11] A. M. Tsvelik, Phys. Rev. B 45, 486 (1992).

[12] P. Jordan and E. Wigner, Z. Phys. 47, 631 (1928).

[13] H. A. Bethe, Z. Phys. 71, 205 (1931).

[14] L. D. Faddeev and L. A. Takhtajan, Phys. Lett. A 85, 375 (1981).

[15] A. Luther and I. Peschel, Phys. Rev. B 12, 3908 (1975).

[16] F. D. M. Haldane, Phys. Rev. Lett. 45, 1358 (1980).

[17] H. Bergknoff and H. B. Thacker, Phys. Rev. D 19, 3666 (1979).

[18] M. C. Cross and D. S. Fisher, Phys. Rev. B 19, 402 (1979).

[19] See also: F. D. M. Haldane, Phys. Rev. B 25, 4925 (1982); I. Affleck, Nucl. Phys. B 265 [FS15], 448 (1986).

[20] A. B. Harris, Phys. Rev. B 7, 3166 (1973).

[21] Z. G. Soos, S. Kuwajima, and J. E. Mihalick, Phys. Rev. B 32, 3124 (1985).

[22] G. Spronken, B. Fourcade, and Y. Lépine, Phys. Rev. B 33, 1886 (1986).

[23] G. Müller, H. Thomas, H. Beck, and J. C. Bonner, Phys. Rev. B 24, 1429 (1981); H. J. Schulz, Phys. Rev. B 34, 6372 (1986).

[24] P. Fulde, Electron Correlations in Molecules and Solids, Vol. 100 of Solid State Sciences (Springer-Verlag, Berlin, 1993).

[25] J. C. Bonner and H. W. J. Blöte, Phys. Rev. B 25, 6959 (1982). 